\title{
Four dimensional calorimetry with both-side readout of the CsI calorimeter in the $K_{L} \rightarrow \pi^{0} v \bar{v}$ search
}

\author{
Katsushige Kotera*† \\ Department of Physics, Osaka University \\ E-mail: coterra@champ.hep.sci.osaka-u.ac.jp
}

\begin{abstract}
We established a method to distinguish photons and neutrons in the calorimeter for the KOTO experiment which studies a rare neutral-kaon decay $K_{L} \rightarrow \pi^{0} v \bar{v}$. The method uses timing information from both front and rear ends of cesium iodide crystals used in the calorimeter. We expect that this new method, combined with the analysis techniques, will suppress the neutron background to be less than 0.2 events when the sensitivity reaches the level of Standard Model prediction.
\end{abstract}

ICHEP 2018, International conference on High Energy Physics

4-11 July 2018

Seoul, Korea

${ }^{*}$ Speaker.

${ }^{\dagger}$ for the J-PARC E14 KOTO Collaboration. 


\section{Introduction}

The aim of the KOTO experiment [1] is to study a rare neutral-kaon decay $K_{L} \rightarrow \pi^{0} v \bar{v}$. This decay is a CP violating process and is sensitive to new physics beyond the Standard Model (SM), because the branching fraction predicted by the SM is small, $3 \times 10^{-11}$, and some theoretical models predict higher branching fractions. The signature of the decay is two photons from the $\pi^{0}$ decay with no other observable particles. Figure 1-a) shows the KOTO detector with the coordinate system used in this article. The decay volume shown in the figure is hermetically surrounded by the calorimeter and veto counters. The calorimeter measures the energies and positions of the two photons from $\pi^{0}$, and veto counters detect extra particles from the background events. Figure 1 - b) shows the front view of the calorimeter which consists of 2716 undoped cesium iodide (CsI) crystals stacked inside the $1.9 \mathrm{~m}$ diameter cylinder. The central $1.2 \times 1.2 \mathrm{~m}^{2}$ region is filled with 2240 of $25 \times 25 \times 500 \mathrm{~mm}^{3}$ crystals (small crystals), and the outer region is filled with 476 of $50 \times 50 \times 500 \mathrm{~mm}^{3}$ crystals (large crystals). Each crystal is read out with a photo-multiplier tube (PMT) attached on its rear end.

a)

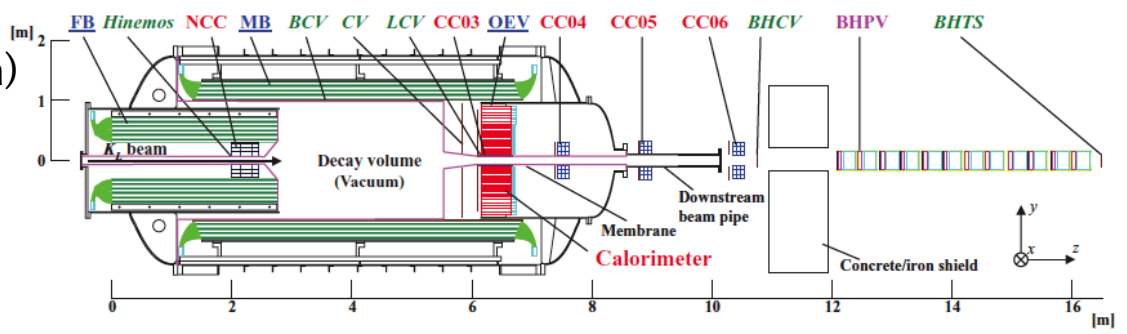

b)

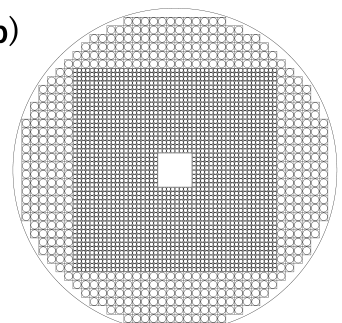

Figure 1: a) Cut-out view of the KOTO detector. b) Front view of the CsI calorimeter.

One of the main backgrounds is caused by neutrons directly hitting the calorimeter and making two shower clusters. Such events are rejected if the calorimeter can distinguish photons and neutrons ( $\gamma / \mathrm{n}$ separation). We have already implemented three analysis methods to reduce this background using the differences between photons and neutrons in 1) two-dimensional $(x, y)$ shapes of clusters in the calorimeter, 2) shapes of waveforms in the readout, and 3) timing of clusters, in which the timing of the second cluster created by a neutron traveling from the initial hadronic shower would be later.

Further reduction of this background by a factor of ten is required when the sensitivity reaches the SM prediction. Thus, we plan to upgrade the calorimeter by measuring their shower depths in $z$; while photons make showers in a shallow region, neutrons can make showers at any depths. To measure the shower depths, we will attach silicon photomultipliers (SiPMs) on the front surface of the CsI crystals and measure the timing difference between the SiPMs and PMTs.

\section{Gluing MPPCs on CsI crystals and their readout}

We use multi-pixel photon counters (MPPC) produced by Hamamatsu K.K. (S13360-6050CS) as the SiPMs. This MPPC has a $6 \times 6 \mathrm{~mm}^{2}$ sensitive area with a silicone cover which is transparent to the ultraviolet (UV) scintillation light, $310 \mathrm{~nm}$, from the CsI crystals. Because this cover has 
a concave surface, we first glue it on a $0.5 \mathrm{~mm}$-thick flat quartz plate with a silicone glue ${ }^{1}$ before gluing it on the CsI crystal. One MPPC is glued on a $13 \times 13 \mathrm{~mm}^{2}$ quartz plate for a small crystal, and four MPPCs are glued on a $25 \times 25 \mathrm{~mm}^{2}$ quartz plate for a large crystal. Quartz plates with MPPCs are glued on crystals with epoxy-type glue ${ }^{2}$ whose transparency is larger than $90 \%$ to UV light. Figure 2 shows the MPPCs on the quartz plates. When gluing the quartz plate, we kept a $0.1 \mathrm{~mm}$ gap between the MPPC and the quartz plate and filled the gap with the silicone glue. After the silicone glue was cured, we applied $2 \mathrm{~N}$ on

a)

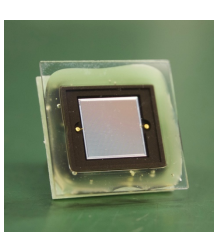

b)

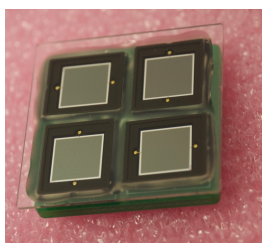

Figure 2: a) Front view of a MPPC on a quartz for a small crystal. b) Front view of four MPPCs on a quartz for a large crystal with a baseboard (green color). the MPPC to compress the silicone, and applied epoxy glue around the MPPC to keep the compressed state. The applied internal pressure prevents cavities between MPPCs and quartz induced by a volumetric strain with the change of temperature.

The number of readout channels is reduced by merging the signals from MPPCs while keeping the performance of $\gamma / \mathrm{n}$ separation. This merging also reduces the amount of material on the front surface of the calorimeter. The signals from four MPPCs are merged in a circuitboard behind the MPPCs. The bias voltages

for MPPCs are supplied in parallel whereas the high frequency signals are read out in series with this circuit [2]. With the parallel connection for applying the bias voltages, the same voltage can be applied even if the dark currents of MPPCs increase due to irradiations with neutrons during the experiment ${ }^{3}$. The series connection for signals keeps small decay constants of the pulse. Four signals from these four merged MPPC signals are summed with an amplifier. Each pulse is smeared with a Bessel filter, and its waveform is recorded in an 8 ns-interval [4].

\section{Expected performance and evaluation from beam tests with a prototype}

We have evaluated the $\gamma / \mathrm{n}$ separation capability with a prototype calorimeter in neutral beams at the Research Center for Nuclear Physics of Osaka University [5]. Photons and neutrons were identified by a time-of-flight technique. The prototype calorimeter was $2 \times 2$ four small crystals surrounded by eight large crystals. Figure 3 shows typical distributions of cluster depths measured with an energy-weighted mean of the timing differences between MPPC and PMT $(\Delta t)$. The timing of each pulse is defined as the time in which the leading edge of the pulse becomes the half of its peak height. The valley in the neutron distribution is caused by a connection of two $250 \mathrm{~mm}$-long crystals to form a $500 \mathrm{~mm}$-long crystal used in the calorimeter. A Monte Carlo (MC) simulation study based on GEANT 4 reproduced this qualitatively. By selecting the events shown with an arrow in Fig.3 90\% of photons survived and 66\% of neutrons were rejected.

\footnotetext{
${ }^{1}$ TSE3032 provided by the Momentive Performance Materials Inc.

${ }^{2}$ EPO-TEK 305 provided by the EPOXY TECHNOLOGY, Inc.

${ }^{3}$ The calorimeter will be exposed to $1 \times 10_{n_{1 M e V}}^{9} / \mathrm{cm}^{2}$ of neutrons at maximum during the KOTO running. We prepared MPPCs irradiated with neutrons equivalent to 1.5 times of the maximum irradiation; the dark current increased by a factor of 100 with the irradiation. The different exposures depending on the location of MPPCs lead to different increases of the dark current. In such a case, the bias voltages for MPPCs in a series connection has to be changed. Thus, the parallel connection of bias is required. We confirmed that the timing resolution unchanded even after the irradiation [3].
} 


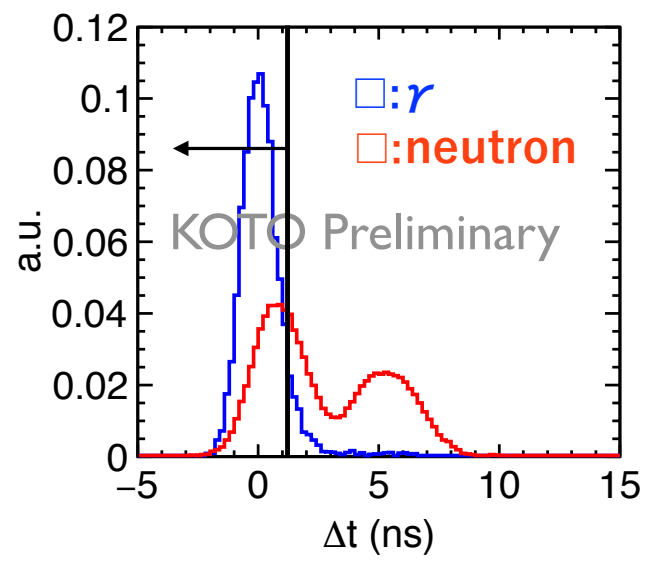

Figure 3: Distributions of cluster positions measured with the $\Delta t$ of photons (blue) and neutrons (red).

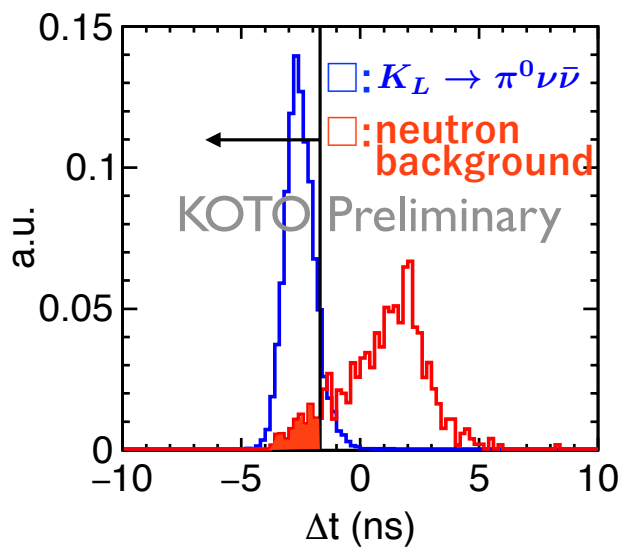

Figure 4: Distributions of $\Delta t$ of a cluster for the signal events (blue) and for the background events (red).

The MC study also estimated the rejection power of this method on the neutron background in the KOTO calorimeter. Figure 4 shows the expected distributions of larger $\Delta t$ of two clusters in the $K_{L} \rightarrow \pi^{0} v \bar{v}$ decays and in the background events. In the background events a primary neutron produced a shower with a hadronic interaction, and a neutron produced in the interaction traveled to another place in the calorimeter, creating a second shower. The distributions were made with events after applying the cluster shape cut and global timing cut; independence on the performance of this method from the pulse shape cut was confirmed in the $\gamma / \mathrm{n}$ separation test. The background events were suppressed to $(8.8 \pm 1.3) \%$ with a $\Delta t$ requirement shown in Fig.4] while keeping $90 \%$ of signal events as expected.

\section{Prospects and status of installation}

In this conference we reported a new upper limit on the branching fraction of the $K_{L} \rightarrow \pi^{0} v \bar{v}$ decay, $3.0 \times 10^{-9}$ at the $90 \%$ confidence level [6]. The neutron background which we discussed in this article was $0.24 \pm 0.17$ events in [6]. The level of this background with the sensitivity of the SM prediction is estimated to be suppressed to the same level as in [6] with this calorimeter-upgrade, a factor of ten, and the improved analysis methods, a factor of three.

We have established the MPPC gluing method on the crystal. We will finish the upgrade by the end of 2018, and resume the physics runs in spring 2019.

\section{References}

[1] J. K. Ahn et al., (J-PARC KOTO Collaboration), PTEP 2017, 021C01 (2017).

[2] A.M. Baldini et al., Eur. Phys. J. C, 78: 380 (2018).

[3] T. Mari, Conference proceedings of ICHEP 2018, Seoul, Korea (2018).

[4] S. Su et al., IEEE Transactions on Nuclear Science 64: 6 , (2017).

[5] H. Nishimiya, Master thesis, Osaka University (2018).

[6] K. Shiomi, Conference proceedings of ICHEP 2018, Seoul, Korea (2018). 